LA-UR-09- OK/38

Approved for public release;

distribution is unlimited.
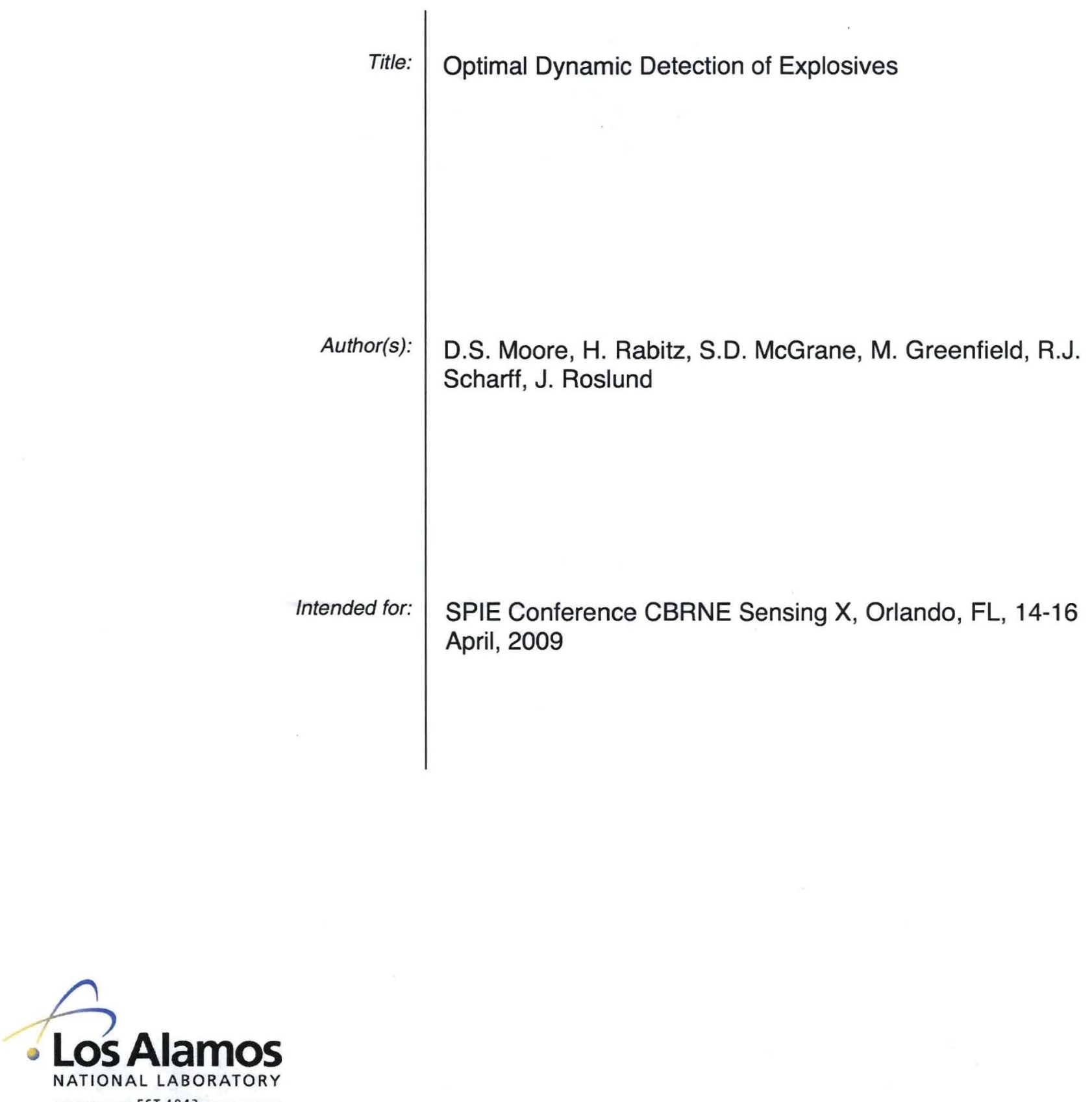

EST.1943

Los Alamos National Laboratory, an affirmative action/equal opportunity employer, is operated by the Los Alamos National Security, LLC for the National Nuclear Security Administration of the U.S. Department of Energy under contract DE-AC52-06NA25396. By acceptance of this article, the publisher recognizes that the U.S. Government retains a nonexclusive, royalty-free license to publish or reproduce the published form of this contribution, or to allow others to do so, for U.S. Government purposes. Los Alamos National Laboratory requests that the publisher identify this article as work performed under the auspices of the U.S. Department of Energy. Los Alamos National Laboratory strongly supports academic freedom and a researcher's right to publish; as an institution, however, the Laboratory does not endorse the viewpoint of a publication or guarantee its technical correctness. 


\title{
Optimal Dynamic Detection of Explosives
}

\author{
D.S. Moore ${ }^{a}$, Herschel Rabitz ${ }^{b}$, S.D. McGrane ${ }^{a}$, M. Greenfield ${ }^{a}$, R.J. Scharff ${ }^{a}$, J. Roslund ${ }^{b}$ \\ ${ }^{a}$ Shock and Detonation Physics Group, Los Alamos National Laboratory \\ ${ }^{b}$ Department of Chemistry, Princeton University
}

\begin{abstract}
The detection of explosives is a notoriously difficult problem, especially at stand-off distances, due to their (generally) low vapor pressure, environmental and matrix interferences, and packaging. We are exploring optimal dynamic detection to exploit the best capabilities of recent advances in laser technology and recent discoveries in optimal shaping of laser pulses for control of molecular processes to significantly enhance the standoff detection of explosives. The core of the ODD-Ex technique is the introduction of optimally shaped laser pulses to simultaneously enhance sensitivity to explosives signatures while reducing the influence of noise and the signals from background interferents in the field. These goals are being addressed by operating in an optimal non-linear fashion, typically with a single shaped laser pulse inherently containing within it coherently locked control and probe sub-pulses. With sufficient bandwidth, the technique is capable of intrinsically providing orthogonal broad spectral information for data fusion, all from a single optimal pulse.
\end{abstract}

Keywords: coherent control, dynamic non-linear, ultrafast lasers, closed-loop optimization, detection, explosives

\section{INTRODUCTION}

The inventiveness and creativity of those that would do the civilized world harm are seemingly limitless. This fact has been true throughout history; today is no exception. While civilized people might have difficulty understanding their enemies' motivation, they can and must use their own creativity to proactively conceive adequate defenses. The most recent alarming increase in number and violence of terrorist bombings has made the task of stand-off detection of improvised explosive devices extremely urgent. Nevertheless, progress to date has been slow because stand-off detection methods must deal with issues of (a) only trace levels of observable material due to low explosives vapor pressures and/or small quantities of surface absorbed explosive residues, (b) excitation of the sample at a distance and return of the resultant radiative signature back to the detector, (c) operation in the presence of atmospheric turbulence and particulate matter, (d) demands of high sensitivity and high signal-to-noise and (e) the need for high selectivity in the presence of possibly very similar but benign background chemicals. Even before the recent alarm, nearly all, if not all, known instrumental methods have already been investigated for their applicability to the detection of explosive materials and none of the explored methods is completely suitable to the task [1-6]. It has been suggested that the most viable approach to standoff detection of explosives is through laser probing [4]. However, a large number of laser based spectroscopic techniques with many variations and subtleties have been explored to date, none of which is even close to completely solving the problem. We believe there is a largely unexplored realm of methodologies based on optimal coherent control that has significant promise in this fight and have begun their exploration.

The new technique of Optimal Dynamic Detection of Explosives (ODD-Ex) is based on the emerging capability to control quantum molecular dynamics phenomena with advanced laser pulse shaping combined with high throughput adaptive experiments guided by special algorithms [7]. By operating adaptively, ODD-Ex seeks out the best laser pulse shapes for the most sensitive and selective explosive spectral signatures. The resultant interrogating laser pulse is specifically shaped to create a maximum signal from explosives while minimizing the signals from background interferents. A key goal of the research is to achieve optimal detection performance by fully exploiting the flexibility of ultrafast laser pulse shaping technology.

* moored@lanl.gov, phone +1-505-665-6089, fax +1-505-667-6372 
The development of ODD-Ex rides on scientific and technological advances with roots going back to the beginning of laser science in the 1960's when the dream of controlling molecular dynamics phenomena was first expressed. A long period of development ensued and significant successful control experiments occurred in 1997 [8]. Their number is rapidly growing with illustrations presently including: (i) sculpting of atomic wave packets, (ii) controlling vibrational motion, (iii) manipulating complex spectra, (iv) selective molecular bond breaking and rearrangements, (v) controlled enhancement of high harmonic radiation, $(v i)$ creating ultrafast solid state optical switches, (vii) managing decoherence processes, (viii) manipulating energy transfer in biological complexes (ix) selectively controlling similar molecules in a mixture, and $(x)$ optimal surface ablation. The common linkage in all of these diverse experiments is their general operational procedure [7], which is based on closed-loop adaptive quantum control techniques as shown in Fig. 1 for detection. By operating under closed-loop it is possible to find shaped laser pulses acting as "photonic reagents" to steer about molecular scale events rather at will, and it is this capability we exploit for the development of high quality ODDEx detectors.

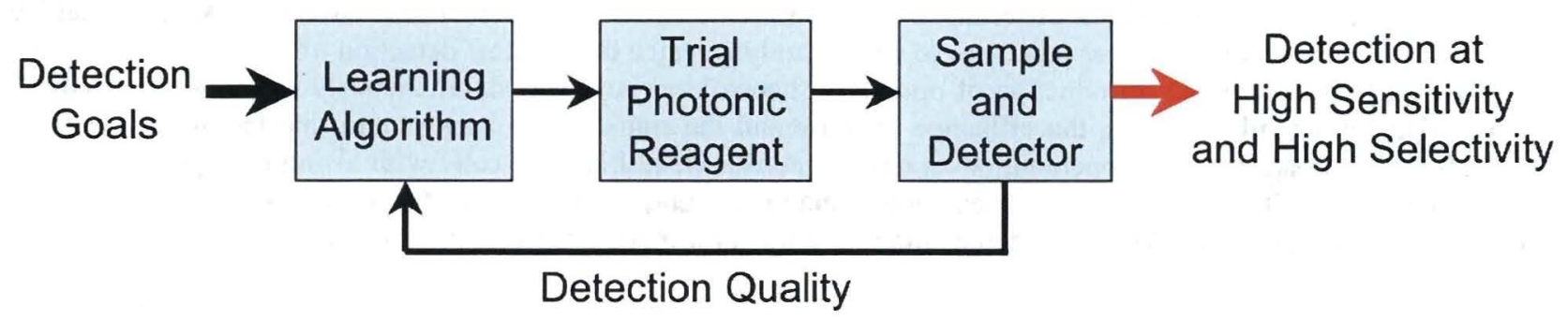

Fig. 1. Closed-loop Adaptive Apparatus for Deducing ODD-Ex Photonic Reagents. The components constitute an adaptive apparatus for molecular optimal control experiments. The apparatus seeks out the best-shaped laser pulses (Photonic Reagents) for the detection of explosives. By operating in this fashion, effective photonic reagent controls can be discovered despite the common lack of a detailed physical model for the explosives or interferents. The technique has been successfully applied to a wide variety of similar molecular scale control goals [7].

\section{THEORETICAL FOUNDATIONS}

The fundamental operating principle of ODD-Ex is to adaptively learn how to non-linearly tailor the excitation of a targeted molecule using intense shaped laser pulses with an apparatus sketched in Fig. 1. The resultant spectrally emitted signature from the tailored excitation can form a unique fingerprint of a molecule while also directing the signature to be as far as possible above the noise as well as distinct from background clutter. These attractive features of ODD-Ex arise from the special ability of the tailored fs pulses to interact with explosive molecules dynamically on their natural time scales. In this context, ODD-Ex can be viewed as an active means of laser detection with tailored photonic reagents, while all prior conventional methods are passive by operating with raw unshaped nascent laser pulses. The development of ODD-Ex to its fullest capabilities holds out the promise of creating a new generation of non-linear high quality explosives detectors.

A rigorous theoretical foundation exists for ODD-Ex based on the fact that a sample mixture of molecules will likely be fully controllable, thereby implying that some suitable laser pulse exists which is capable of creating a maximum discriminating signature from the desired species [9]. Utilizing this principle provides the framework to exploit advanced ultrafast laser techniques, including broad bandwidth high resolution shaping of the pulses. The resultant photonic reagents can interact strongly with a sample to create a tailored molecular excitation that, in turn, can produce a unique spectrally emitted signature from an explosive or other molecule targeted for detection. The general operating mechanism of ODD-Ex is illustrated in Fig. 2. The flexibility of ODD-Ex, offered by its adaptive closed-loop operation in Fig. 1, should open up the ability to optimize performance from existing non-linear spectroscopic techniques, but most importantly, ODD-Ex can go beyond existing spectroscopies to seek the best laser control pulse shape and resultant spectral signature for detection. The execution of ODD-Ex in the field should be rapid. The complete development of ODD-Ex will push the technique to its fullest capabilities while also being mindful of safety issues for ultimate field applications in a domestic environment. 

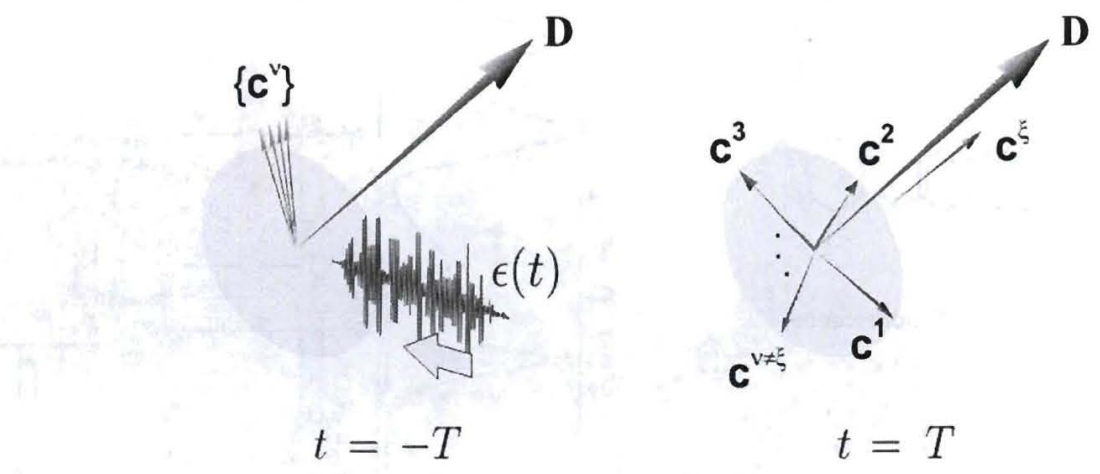

Fig. 2. Graphical representation of the ODD-Ex mechanism. Initially at $t=-T$ the vectors $\mathrm{c}^{v}=1,2, \ldots$, representing the state of each of the explosive and bakground quantum systems, are very similar. After the optimal control pulse $\varepsilon(t)$ interacts dynamically with the mix of systems, the selected vector $\mathrm{c}^{\mathrm{s}}$ for the explosive is aligned parallel to $\mathrm{D}$, the detection state. The vectors of the background species $(v \neq \xi)$ are rotated to be perpendicular to D to diminish their interfering signals. Operating in this fashion can enhance signal-to-noise and selectively.

As an example of the ODD technique, it was able to discriminate between two exceedingly similar flavin molecules (RBF and FMN) that have virtually identical electronic absorption and emission spectra [10]. Nevertheless, by introducing a suitable shaped photonic reagent, deduced by the procedure in Fig. 1, the spectral emission signatures of these two molecules could be dramatically drawn apart for their simultaneous detection in a mixture (see Fig. 3). The detection mechanism was demonstrated to involve controlled coherent quantum dynamics in keeping with the conceptual perspective in Fig. 2 showing that maximum detection performance can be attained when operating in this domain. The extreme selectivity demonstrated in Fig. 3 also opens up the prospects for ODD-Ex to detect and distinguish the presence of explosive fragments of partially reacted materials as well as the nascent explosive molecules.

For nearly twenty years, the Rabitz group at Princeton has been the developer of many of the key principles of quantum control including its theoretical foundations, modeling of the concepts, and most recently, serving as director of an ultrafast laser laboratory for implementation of quantum control especially for purposes of chemical agent detection. This latter work has also involved demonstrations of how suitable non-linear controls can be utilized to enhance signalto-noise [11] while simultaneously achieving excellent selectivity. The general field of controlling quantum molecular dynamics phenomena is now at an advanced stage of development with activities in many laboratories around the world including at Princeton and Los Alamos.

\subsection{Theory}

The basic theoretical foundations for explosive agent detection are being explored based on the principles of (a) optimally induced quantum dynamics for the creation of a highly selective signature and (b) the non-linear enhancement of signal-to-noise by both driving up the signal intensity as well as using the dynamics of the explosive agent itself as a filter to diminish the impact of noise. In addition, the ability to create special laser pulse shapes to enhance the robustness to noise will be analyzed. The quantum mechanical and mathematical formulations of these theoretical concepts will be developed by building on current general theory for controlling quantum phenomena.

\subsection{Modeling and Simulations}

Building on the theory, specific models are being developed to explore the key concepts. The goal is to operate with models that are as simple as possible while still retaining the essential physical and chemical features for explosive detection. These modeling efforts assess the ability of broad bandwidth ODD-Ex to create spectrally rich orthogonal explosive signatures for data fusion and more reliable detection. In addition the simulations explore the ability to manage high quality detection operations even with atmospheric distortion of the propagating shaped laser pulse while optimally enhancing the return signal. The results of the simulations will be used as feedback to refine the theory of ODD-Ex for guidance on how to attain maximum laboratory performance from the technique. 


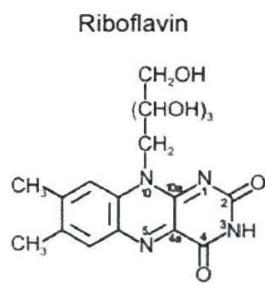

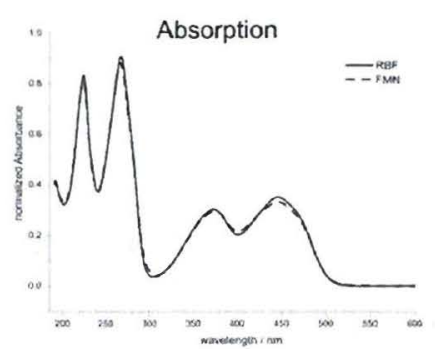

Flavin Mononucleotide<smiles>Cc1cc2nc3c(=O)[nH]c(=O)nc-3n(CC(C)O)c2cc1C</smiles>
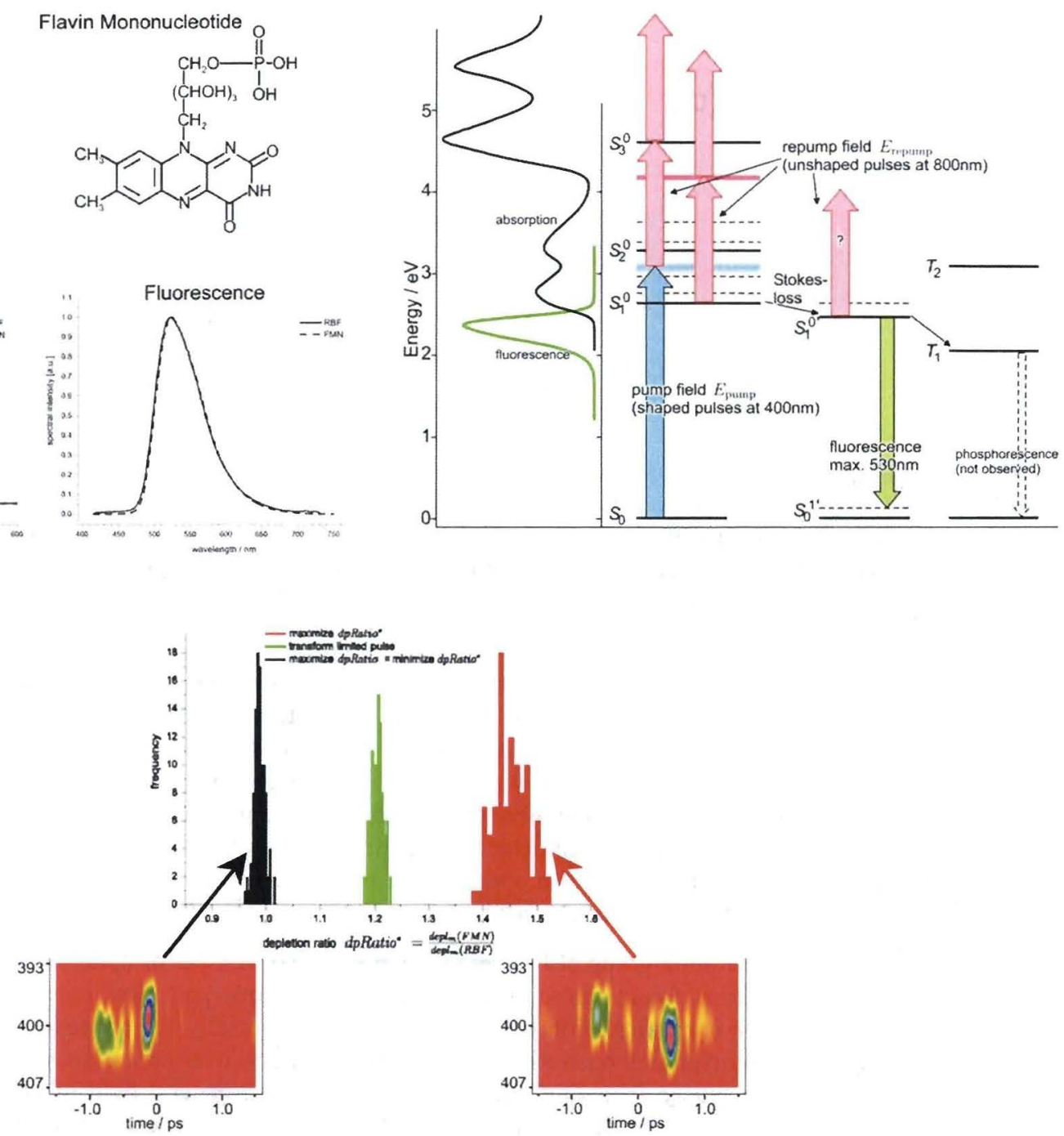

Fig. 3. ODD-Ex concepts illustrated using riboflavin (RBF)) and flavin mononuclide (FMN). Top left: the absorption and emission spectra of RBF and FMN are nearly identical, making them nearly impossible to discriminate by conventional spectroscopic tools; top right: one form of ODD (i.e., spectra depletion). The shaped control pulse is deduced optimally using adaptive closed-loop control techniques; bottom: The data shows the ability of suitably shaped laser pulses to either enhance or diminish the signal ratio (spectral depletion signal) RBF/FMN. The two distinct laser pulses accomplishing the discrimination are shown in the lower portion of the figure as wavelength/time plots.

\section{EXPERIMENTS}

The experiments explore the key ODD-Ex concepts emanating from the theory and simulations. These laboratory demonstrations particularly focus on enhancing selectivity and signal-to-noise, especially by exploiting the non-linear capabilities of intense shaped photonic reagents. The ability of ODD-Ex to optimally utilize laser resources is critical when working with very low concentrations of explosive vapors in air with the goal of assessing the smallest concentrations that may be reliably detected. This facet of ODD-Ex is aimed at improving limits of detection.

The overall performance of ODD-Ex will be characterized by ROC curves to identify interrogating laser pulses that maximize sensitivity while reducing false alarm rates. The inherent broad bandwidth orthogonal data generated by ODD-Ex should greatly facilitate this task. All of the theoretical and laboratory efforts will build on prior experience in these areas at Princeton and in the laboratories at Los Alamos. In addition, extensive collaborations with many other 
theoretical and laboratory programs in the general domains of quantum control and explosives are being drawn upon to achieve the ultimate aims of ODD-Ex.

\section{Optimal Dynamic Detection of Explosives (ODD) Protocol}

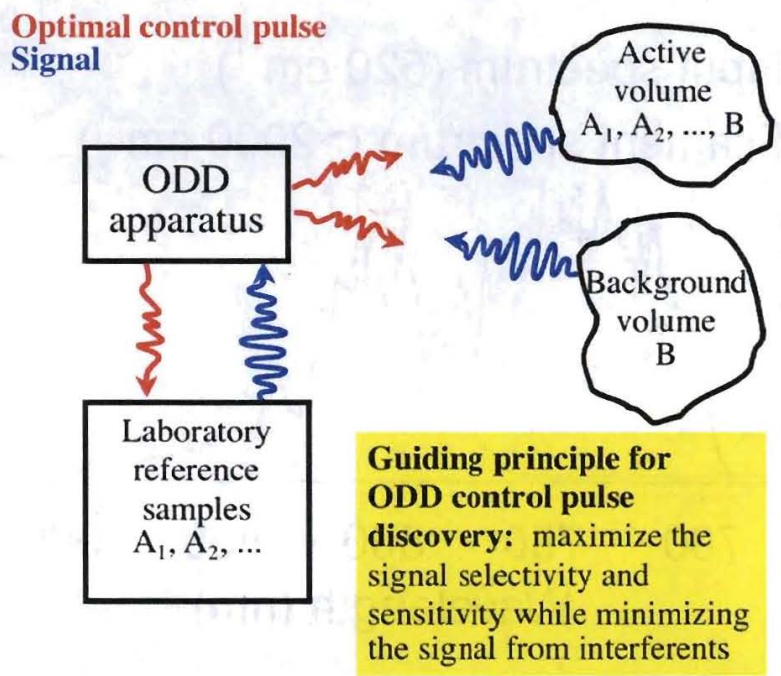

Fig. 4. Optimal Dynamic Detection of Explosives protocol. The guiding principle for discovery of the optimal control pulse is to maximize the signal selectivity and sensitivity while minimizing the signal from interferents.

Today's commercial ultrafast laser systems are quite capable of producing stable trains of $35 \mathrm{fs}$ length pulses with a few $\mathrm{mJ}$ energy per pulse at a few $\mathrm{kHz}$ repetition rate. The wavelength bandwidth of these pulses is of the order of $40 \mathrm{~nm}$ (time/bandwidth product assuming transform limit), which at $800 \mathrm{~nm}$ central wavelength is only $5 \%$. In other words, the wavenumber bandwidth at $800 \mathrm{~nm}$ of such pulses is only $\sim 550 \mathrm{~cm}^{-1}$. Rabitz et al. have shown that the control landscape has no traps given sufficient laser resources [12], and also that insufficient resources leads to a family of control solutions known as level sets [13]. These level sets can be thought of similarly to the equal-elevation lines on a topographical map. Although they represent optimized solutions, they aren't at the peak of the control landscape because of insufficient laser resources. We have found that control experiments using the $100 \mathrm{fs}$ output of our commercial laser system is insufficient to completely optimize the control of a chemical reaction (stilbene isomerization) [14], giving instead a family of solutions of quite similar product yield ratio improvement.

Therefore, we are exploring methods to broaden the bandwidth of these laser systems. These schemes include noncollinear optical parametric amplification (NOPA) $[15,16]$ and self-phase modulation (SPM) in noble gases either as a free filament or confined in hollow-core fibers [17]. The NOPA concept was aided by the discovery of a "magic" phase matching condition in type I beta-barium borate (BBO) nonlinear crystals pumped at $400 \mathrm{~nm}$ (the second harmonic of the Ti:sapphire fundamental wavelength). At $400 \mathrm{~nm}$, the pump inclination to the direction of the seed matches almost perfectly the angle of birefringent walk-off between the ordinary and extraordinary waves inside the crystal, allowing $\mathrm{BBO}$ path lengths as long as 1 to $2 \mathrm{~mm}$ even in 5-fs NOPAs, resulting in huge conversion efficiencies. Further improvement of the phase matching bandwidth in NOPAs can be achieved by increasing the spectral width of the pump and adjusting frequency-dependent pump angles [15]. The filamentation band broadening scheme is shown in Figure 5 along with an example of the bandwidth achievable. To date, that bandwidth is of the order of $\sim 2000 \mathrm{~cm}^{-1}$, covering a large portion of the vibronic envelope within accessible electronic transitions of explosive molecules. 


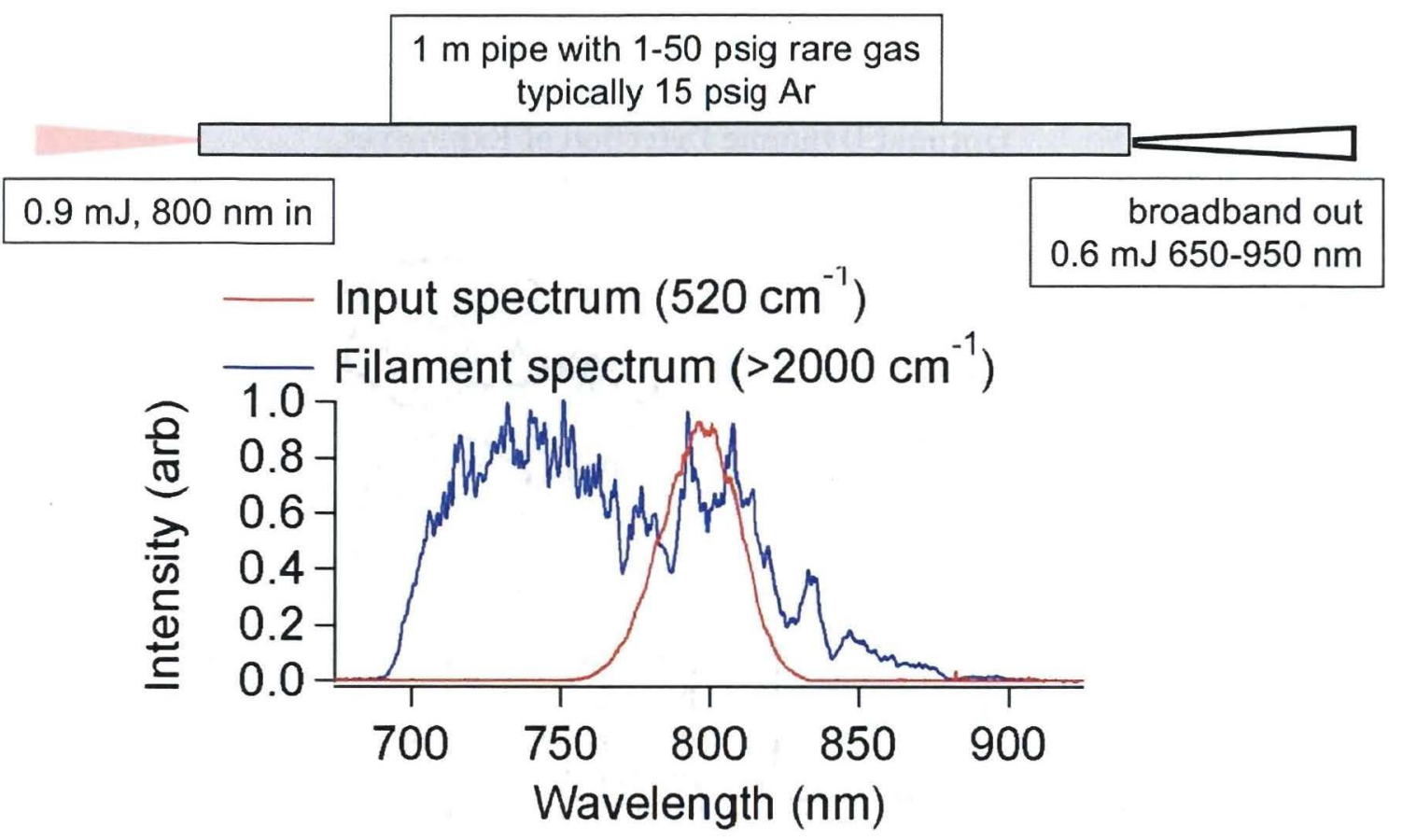

Fig. 5. Filamentation band broadening scheme using a pressurized Ar filled tube and $35 \mathrm{fs}$ input pulses of $\sim 1 \mathrm{~mJ}$ energy.

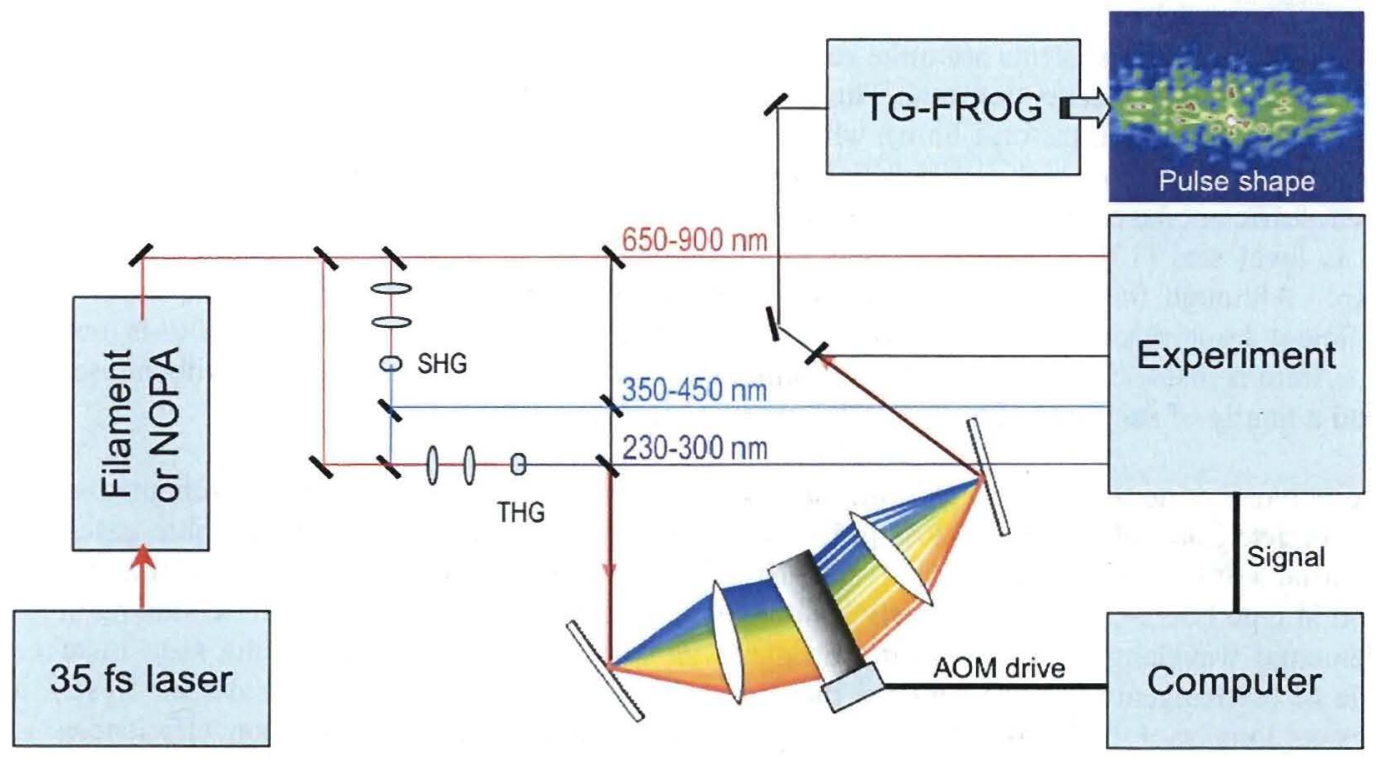

Fig. 6. General scheme for ODD-Ex using presently available laser resources, allowing for a wide variety of spectroscopic control and probe schemes. Also shown is a transient grating frequency resolved optical gating (TG-FROG) component to measure the pulse shape.

A schematic of an instrument designed to explore a variety of single and multiple pulse spectroscopic methods for their capabilities to be optimized for explosives detection is shown in Figure 6. Ideally, the control and probe portions of the photonic reagent would be contained within a single shaped laser pulse. While that is the ultimate goal, a variety of other kinds of spectroscopic tools are being investigated along the way, such as coherent anti-Stokes Raman, stimulated 
emission, and others, because the available bandwidth, even given our new broadening tools, may still be insufficient to achieve the ultimate optimized detection limits.

\section{OUTLOOK}

We are only beginning to scratch the surface of what optimal coherent control methods can do for the detection of explosive traces and vapors. Already simple pulse shaping (using practitioners intuition rather than closed loop optimization) has been shown capable of drastically reducing the non-resonant background in coherent Raman spectra $[18,19]$ and enabling detection of molecules at a distance using non-linear spectroscopies [20]. There has been a recent application of adaptive pulse shaping towards selective CARS excitation [21]. We are optimizing these and other dynamic spectroscopic methods using closed loop methods, which will result in large further improvements in sensitivity and selectivity. The ultimate goal for ODD-Ex is to maximize the sensitivity and selectivity using a single shaped broadband ultrafast laser pulse to both control and probe the signature of a given explosive at stand-off distances. We are at the beginning of a revolution in this area, and are very excited for its future development.

\section{REFERENCES}

1. Yinon J., "Field detection and monitoring of explosives," Trends Anal. Chem. 21, 292-301 (2002)

2. Bruschini C., "ExploStudy, Final Report," Ecole Polytechnique Federale de Lausanne, Web URL: http://diwww.epfl.ch/lami/detec/ (2001).

3. Steinfeld J.I., Wormhoudt J., "Explosives detection: A challenge for physical chemistry," Ann. Rev. Phys. Chem. 49, 203-32 (1998)

4. "Existing and potential Standoff Explosives Detection Techniques," National Research Council, National Academies Press (2004).

5. Moore D.S., "Instrumentation for trace detection of explosives," Rev. Sci. Instr. 75, 2499-2512 (2004)

6. Moore D.S., "Recent Advances in Trace Explosives Detection Instrumentation," Sens. Imaging 8, 9-18 (2007)

7. Walmsley I., Rabitz H., "Quantum Physics Under Control," Physics Today, 56, $43-49$ (2003).

8. Rice S.A., Manz J., Kohler B., Mukamel S., Brumer P.W., et al. "Perspectives on the control of quantum many-body dynamics: Application to chemical reactions," Adv. Chem Phys. 101, 213-283 (1997)

9. Turinici G., Ramakrishna V., Li B., and Rabitz H., "Optimal Discrimination of Multiple Quantum Systems: Controllability Analysis," J. Phys. A, 37, 273-282 (2004).

10. Roth M., Guyon L., Boutou V., Courvoisier F., Roslund J., Wolf J.-P., Rabitz H., Optimal dynamic discrimination of similar molecules in the laboratory, (in preparation).

11. Bartelt A., Roth M., Mehendale M., Rabitz H., "Assuring Robustness to Noise in Optimal Control Experiments," Phys. Rev. A, 71, 0633806 (2005).

12. Rabitz H.A., Hsieh M.M., Rosenthal C.M., "Quantum Optimally Controlled Transition Landscapes," Science 303, 1998-2001, (2004)

13. Roslund J., Roth M., Rabitz H.A., "Laboratory observation of quantum control level sets," Phys. Rev. A 74, 043414 (2006)

14. Greenfield M.T., McGrane S.D., Moore D.S., "Control of cis-stilbene photochemistry using shaped ultraviolet pulses," J. Phys. Chem. A. DOI: 10.1021/jp801758v (2009)

15. Baltuska A., Kobayashi T., "Adaptive shaping of two-cycle visible pulses using a flexible mirror," Appl. Phys. B 75, 427-443, (2002)

16. Driscoll TJ, Gale GM, Hache F, Ti:sapphire second-harmonic-pumped visible range femtosecond optical parametric oscillator," Opt. Commun. 110, 638-644 (1994)

17. Nisoli M., Stagira S., De Silverstri S., Svelto O., Sartania S., Cheng Z., Lenzner M., Spielmann Ch., Krausz F., "A novel-high energy pulse compression system: generation of multigigawatt sub-5-fs pulses," Appl. Phys. B 65, 189196, (1997)

18. Yellampalle B., Averitt R.D., Efimov A., Taylor A.J., "Spectral interferometric coherent Raman imaging," Opt. Express 13, 7672-7682, (2005)

19. Pestov D., Murawski R.K., Ariunbold G.O., Wang X., Zhi M.-C., et al., "Optimizing the laser-pulse configuration for coherent Raman spectroscopy," Science 316, 265-268, (2007) 
20. Katz O., Natan A., Silberberg Y., Rosenwaks S., "Standoff detection of trace amounts of solids by nonlinear Raman spectroscopy using shaped femtosecond pulses," Appl. Phys. Lett. 92, 171116, (2008)

21. Zhang S., Zhang L., Zhang X.-G., Ding L.-T., Chen G.-L., Sun Z.-R., Wang Z.-G., "Selective excitation of CARS by adaptive pulse shaping based on genetic algorithm," Chem. Phys. Letters 433, 416-421, (2007) 